\title{
Research Analysis on the Implementation of Mobile Wallet under E- commerce Platform
}

\author{
Yi Yang, Zhu Mei, Jingdong Liu and Xing Wang \\ Building and Technology Engineering company \\ Physical Education Department \\ School of Design \& Art \\ International School \\ Shenyang Jianzhu University, Shenyang China \\ yangyi@sjzu.edu.cn,mz@qq.com, liujd@sjzu.edu.cn, wangxing@sjzu.edu.cn
}

Keywords: Mobile wallet; e-commerce; SWP; RFID

\begin{abstract}
With the rapid development of Internet technology, E-commerce is increasingly accepted by the people. E-commerce transactions via the Internet to achieve a convenient, with ubiquitous business features. But such business model by the trading range of restrictions, has the deficiency of movement flexibility. It needs to break the traditional business model, and establish a new service model to provide their own value, and to provide users more comfortable and convenient service. According to the principles of system design and system architecture, we put forward a set of electronic payment channel for mobile services, architecture design, implementation and applications in this paper. The full technical program integrated application SWP patch card, dual SIM card interface chip, dual-core dual-interface SIM card, chip card, to achieve a set of efficient, safe, support multiple devices at the same time, to pay more than a new wireless with no physical card-based payment system.
\end{abstract}

\section{Introduction}

With the rapid development of mobile communication technology, mobile phone has become a necessity to carry the majority of users. In daily life, people can make a variety of payment activities [1]. With the emergence of mobile payment services, it can be done by phone without having to pay cash or credit card, to the majority of people's daily life convenient. Mobile payment services as a new method of payment, with factors in convenience, fastness, safety, etc., is the future trend of mobile e-commerce development, which has a great business prospects, and will make mobile ecommerce and wireless financial se-tor onto a new level.

Mobile payment business is the customer's phone number and bank card account for binding. Mobile phone users choose a bank account as payment of its mobile payment account payments through mobile phones in the future, acting by the designated bank account to facilitate payment. "Mobile wallet" is bound by the only phone number, to establish an independent virtual money account for account management and payment of operating modes, and the user adopts SMS, STK, GPRS, RFID, etc., to pay for remote or on-site [2,3]. While it is common for all kinds of daily life such as transfer, bill payment, transaction, travel, shopping and other applications, "Mobile wallet" service is based on China Mobile developed radio frequency identification (RFID) technology in small business e-wallet payment [4]. After the business user in the process, we can use the phone for China Mobile POS machine merchant services credit card spending. Users get the payment card through STK menu open "mobile wallet" service, can use the phone immediately placed in a special cloth merchant POS machines card for onsite consumption, and to enjoy convenience to pay with random consumption.

"Mobile Wallet" is a comprehensive class of business to pay the various functions of a new service, which is based on bank card account for the financial support, mobile phone tools for the 
transaction of business service model. The user in the user's bank accounts and mobile phones number are bound, via cell phone text messages, IVR, WAP and other means. So the user can bind to operate accounts to achieve shopping, on behalf of the payment, transfer, account balance inquiry and can get the transaction through SMS, etc. to be informed of the results and account change notifications.

\section{Mobile Wallet Business Development Statuses}

Currently the world's mobile operators have launched a variety of "mobile wallet" business plan, that is, the "mobile wallet" for payment and relative technology becomes more mature. In recent years, the global mobile payments increasingly accelerated pace of business development, with mobile operators, banks and other service industries which are all aware of the mobile payment services. It will bring huge economic benefits, brands and social benefits, so we have to seek cross-industry cooperation, to enhance their market competitiveness and service capabilities.

The most mobile payment began in the United States, Japan and South Korea and Europe, which has become so main-stream in many countries and regions. Swedish Paybox Company in Germany, Sweden, Austria, Spain and other countries launch mobile wireless payment system as a tool to replace the traditional credit card. For the service user, as long as the service providers to register and get account there, they buy goods or pay a service fee, and they can provide a mobile number directly to the merchant to pay. So far, the mobile phone in Japan and South Korea has become a mainstream payment devices terminal. Nokia also launched in overseas markets on the phone to support such services, mainly aimed at ages 25-45 years old in the mobile payment ser-vices to consumers, who are the main user groups. South Korean mobile operators mobile payment industry practice is combined with various mobile payment-related medium-sized enterprises co-launched the business, including banks and credit card companies, vending machine manufacturers, retail stores, ATM vendors and taxi companies [5].

South Korean consumers have become accustomed to using the phone as a credit card; Almost all Korean retail banks can provide mobile banking services. There are more than 30 million people who buy a new phone automatically will choose to have a special memory card slot, for storing bank transactions, and transaction information for encryption. In Korea there are thousands of restaurants and shops have read from the phone via infrared terminal credit card information, to meet the customer making purchases by phone. Use of mobile payment "wireless billing service" has become three mobile operators a major business in South Korea with a growing number of mobile phone users who are applying for a credit card smart card-enabled mobile phones. "The phone as a credit card" has become a trend.

In China, China Mobile and China Unicom have been carried out earlier when a pilot mobile payment services. In Shanghai, for the convenience of train users, China Mobile is also preparing to launch year's ticket phone order business, and the business processes and set the other ticket is very similar, so that users do not need to line up tickets, especially in the future railway transport peak. The user will be more easy and convenient, when the mobile is still with the railway authorities to consult on the business. Mobile phone users open "mobile wallet" service, and the user bank account and phone number to be bound. Users can use SMS, voice, GPRS mobile payment methods, etc. to achieve objects [6]. With the development of mobile payment services, mobile payment capabilities more widely, such as pay electricity, water, cable television fees, fee online games, download mobile resource cost, online shopping expenses. Consumers can also use mobile phones to buy drinks from vending machines food, buy metro tickets and tickets by phone to complete online booking and payment, too numerous to be mentioned. China's "mobile wallet" is the main areas including online ticketing, game cards, software, services, Welfare, insurance services, network television and electrical household magazines. "Mobile wallet" services become a major mobile convergence applications development trend, currently confined to micro-payment on future development as well with a large space. At present, mobile banking is only in the initial early stage. For the "mobile wallet" consumer, there are many restrictions, such as network penetration businesses, users of consumer psychology, etc., restricting the "mobile wallet" business development prospects. But with 
the people mature consumer psychology, "mobile wallet" service will accomplish much, in line with the trend of development of $3 \mathrm{G}$ information age. It can make people's lives more convenient, to bring information technology to experience the joy, all this will be more subject to mobile operators, merchants and users to be promoted jointly.

\section{Mobile Wallet Businesses}

Firstly, do the process of moving the NO.1 positive charge. Insert the key frame on the ended position, drag the positive charge to the desired location, and create the traditional interpolation. The scintillation can be made by emptying out a few frames to be blank frames, and then used as the key frame until the end. This is shown in Fig. 12. Similarly, complete the movement, interpolation and maintenance of the positive and negative charges left.

"Mobile wallet" is based on China Mobile network and Internet technology, with the use of mobile tools like SMS, voice, STK, WAP, RFID, etc., to achieve the consumption of mobile payment account, voucher, check, transfer and other variety of e-commerce operation, and related data management services. "Mobile wallet" service is provided by a mobile payment service ability. The user can be physical goods, digital goods and services to buy and pay for value-added data services. "Mobile Wallet" is the operator with banks and other financial sector cooperation, through the user's mobile phone number and bank account (number) bundle, use SMS, WAP, Internet and other mode of operation, completing a mobile payment services, anytime and anywhere, and showing its outstanding advantages. "Mobile wallet" works through the customer's mobile phone number and bank card payment account for efficient binding, China mobile phone has a variety of mobile customers pay channel services.

"Mobile wallet" support specific services including recharge, consumption, composite applications, confiscated cell phone calls, personal account information, pre-paid recharge, buy lottery tickets, purchase IP cards, mobile phone newspaper subscriptions, distance education, public utilities payment, insurance and other mobile phones as a number of businesses. In these operations, the "mobile wallet" is responsible for providing contract business customers to buy the product or service. "Mobile Wallet" payment platform deals with specific payment information. Consumers only needs a cell phone, or we can complete the financial transaction, and enjoy its convenience, while its applications are now generally include recharge, bill payment, electrical appliances purchased, securities investment and online services. Since this payment has not time and space, the advantages of geographical restrictions, has become a cash payment, bank card payment and online payment, etc. The outside of a powerful means payment, and gradually development into a system in an existing financial services kind of a more competitive new service model.

"Mobile Wallet" project is a combination of electronic money, authentication, mobile communications and mobile terminals new business anywhere, to enjoy a variety of convenient services, products and services to enrich the connotation of the parties, while the side to facilitate the efficient and safer way provide customers with existing and innovative products and services. Although the mobile payment market potential is huge, the current mobile payment services in China is still relatively slow pace of development. We analyzed the constraints of mobile payment services directly to the key factors in the following four areas:

- Security issues.

- Consumer spending habits

- Payment terminals

- It has not yet formed a complete industrial chain.

China's mobile payment industry is facing these problems the test, also plagued by other obstacles. However, some issues are not able to solve overnight, step by step. Mobile payment is undoubtedly the future development trend of mobile phones. In particular Near Field Communication (NFC) support this feature as one of the main techniques have begun to be more mobile phone manufacturers focused. The success of Japan and other regions of the tests showed that this technology allows mobile phones to gradually replace credit cards and cash, as tickets and movie tickets to use as office and home of the electronic key. In the "mobile wallet" business development, 
like China and Japan, is indeed very different in domestic situation. First, in a smaller area of Japan, the population is relatively concentrated, the operators can efficiently promote new business; but in the vast and complicated Chinese market, the promotion of each business must have a combination of local market characteristics, to promote the efficiency of the limitation. In addition, the Japanese mobile phones are customized by the operator. They can analyze their own development needs to have the business requirements of mobile phone manufacturers directly embedded with "mobile wallet" function, including software and payment chips, etc., and their phones are relatively simple as the diversification of domestic mobile phone market. It is also decided in the domestic market that can not easily copy the Japanese mobile payment mode. So far, China Mobile has been launched in nine cities across the country a "mobile wallet" service. The advantages such as Beijing linkage which are through service providers, the operators, financial institutions, users of the three linked will form a complete industrial chain.

\section{Mobile Wallet Business Overall Program Design}

The system is divided into mobile banking applications and mobile micro-payment fee of two parts (Due to the payment service can now pay the bank being applied BOSS interface, this paper adopts the mobile micro-payment services for the design and implementation). The main objective is to design systems for mobile terminals which provides multiple access methods, and can be readily extended to access methods without affecting the core platform performance. The number of mobile phone users adapt to the growing demand for mobile phone users who can conduct various transactions providing a safe, fast and easy access. According to our design goals we establish the need to build an integrated platform to control the transaction process that is to be achieved: mobile wallet "business critical, the platform should be provided with accounting system, the mobile communication system mobile SP, banking transactions, communication interface. According to business processes, the business platform can be divided into three layers: the service access layer, business layer and accounts processing system interface layer, the logical structure of the system design are as follows:

(1) Business access layer: responsible for receiving client user's request. The request processes a standard format to the business process layer, and this layer should support and application system authentication and data encryption and decryption.

(2) Business intermediate processing layer: responsible for handling business data, the types of transactions according to the user the appropriate treatment, and completes the major business functions, logic functions. The system management functions and financial management functions are related to various accounts, such as payment, logging, reconciliation, inquiry, transfer, settlement, billing and so on.

(3) Accounting System Interface Layer: transaction data will be forwarded to the operator's business support systems, or banking and financial system to complete the final processing.

Trading platform is a concrete realization of the "mobile wallet" system platforms and SMS Center (SMSC) is connected. We need to establish a special access number mobile wallet and mobile wallet platform to develop the transaction message format. Mobile Wallet platform center via SMS sent to the payment terminal SMS. SMS pay terminal also send SMS messages in the form of center, according to the message on the message center number to identify the target, which is the specific micro-payment messages, and forwarding to the mobile wallet platform. Mobile phone users can use SMS and the required format is directed to a dedicated access number for account inquiries, consumer payments, transfers and other kinds of transactions.

To conduct a transaction, due to the payment account in Mobile BOSS system, mobile wallet platform has a deal with the BOSS system interface. The user authentication and transaction with amount of the transaction query generate and accounts in a single operation by the transaction interface. For the accounts stored by the bank card for the transaction of the user, the "mobile wallet" system platform and banking system interfaces, can be used a proxy server in order to ensure the safety of both systems, based on Socket Communications. To save resources, the sharing of resources can be used to transform the current BOSS system interfaces with the bank. In addition, 
the mobile wallet service platforms and mobile business applications will be developed by SP systems which have a common transaction interface; the interface can be realized through the transaction data.

\section{Conclusions}

"Mobile Wallet" is a phone with a credit card which naturally integrates two high-tech products and it has evolved into a new payment instruments providing safe, convenient and stylish means of payment. Currently supported services include: bill self-pay, prepaid, bill payment, balance inquiry calls, prepaid cell phone wallet, consumer, bank card balance inquiries, composite applications, and so on. Based on the principles of system design and system architecture, we propos a set of services for mobile E-payment channel architecture design, implementation and applications, including the "mobile wallet" card-side business process design, the systems design and use of personal data terminal machine transaction process design, combined with the successful experience of foreign countries presents a practical web-based mobile operators, to provide value-added services that is different from the traditional "mobile wallet" function of the system. The technical program integrated application SWP (Single Wire Protocol) all card, SWP patch card, single-chip dualinterface SIM card, dual SIM cards and dual interface chip cards, to achieve a high efficiency, security. The supported multiple devices at the same time pay more than the new wireless, and there is no physical card-based payment CUP in the system. The "mobile wallet" service system has been developed into products.

With the rapid development of science and technology, "mobile wallet" terminal technology can eliminate the bottleneck. Further in smart phone, the price will drop significantly promoting the "mobile wallet" of rapid development. With the development of future technology, "mobile wallet" technology business will become more perfect in intelligent 3G mobile phones, such as fingerprints, security phone, etc. Onto the market for mobile payments offer a super carrier, mobile phone further intelligent, the prices will fall to promote the "mobile wallet" business. In the $3 \mathrm{G}$ era, high-speed network will provide users with fast and smooth platform for mobile applications. In addition to the income, "mobile wallet", that mobile operators paid to another benefit is making it easier to access the user's behavior information, and establish a complete understanding of user behavior, with other business operators, the introduction and promotion of both large benefits.

\section{Acknowledgment}

This work was supported by Youth Foundation of Shenyang Jianzhu University (NO. 2015031)

\section{References}

[1] Chowdhury Riti, De Debashis. "Secure money transaction in NFC enabled mobile wallet using session based alternative cryptographic techniques" , Communications in Computer and Information Science, vol.245, Sep.2011, pp.314-323.

[2] Ho Debby, Head Milena, Hassanein Khaled. "Developing and validating a scale for perceived usefulness for the mobile wallet", Advances in Intelligent Systems and Computing, vol.206, Jan.2013, pp.469-476.

[3] Mjølsnes Stig Frode, Rong Chunming. "On-line E-wallet system with decentralized credential keepers", Mobile Networks and Applications, vol.8, Mar.2003, pp.87-99.

[4] Kreft Heinz, Adi Wael. "Wallet based e-cash system for secured multi-hop cash exchange", Proc.International Conference on Information and Communication Technologies: From Theory to Applications, Apr.2008, pp.325-329. doi:10.1109/ ICTTA. 2008.4529987.

[5] Carey David. "Turning a mobile phone into a wallet", Electronic Engineering Times, Oct.2006, vol.1443, pp.24-25. 
[6] Weiss Kenneth. "Mobile payments, digital wallets and tunnel vision", Biometric Technology Today, v2011, Oct.2011, pp.8-9 